\title{
A GUIDE TO AVULSION INJURIES
}

\section{By Laura} Crawford

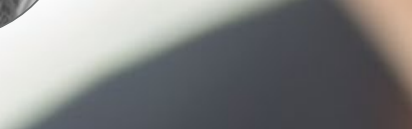

What is an avulsion injury?

The complete displacement of a tooth from its socket. An avulsed permanent tooth is a true dental emergency and in practice you should be prepared to offer immediate advice over the phone and have understanding of the management of such injury.

\section{Emergency information over the} phone for first aid

- Find the tooth, pick it up by the crownavoid touching the root surface

- Wash gently under cold running water for 10 seconds

- Place tooth back in socket and hold in place with clean handkerchief

- If not possible place in suitable storage medium e.g. milk and attend dental practice immediately

It is unlikely that a patient will present with a re-implanted tooth, therefore it is likely you will provide the first stage of management in dental practice.

\section{Patient assessment}

While there is an emphasis on the speedy reimplantation of avulsed teeth it is important to consider the patient as a whole and assess as per any dental trauma.

- Medical history

- Mechanism of injury

- When did the injury occur?

- Where did the injury occur?

- Loss of consciousness

- Previous injury to the tooth?

- Has the injury changed the way you bite?

- Rule out alveolar fractures

- Assess tetanus status
- Extra oral and intra oral examinationincluding assessment of soft tissues for any other injuries

\section{Avulsed Permanent Incisor - \\ Open Apex}

For these you should:

- Administer local anaesthetic

- Irrigate socket with saline

- Reposition

- IOPA radiograph to assess position

- Add a flexible splint for 2-4 weeks if $>60$ minutes extra oral time

- Prescribe antibiotics for seven days (remember check for allergy)

- Recommend chlorohexidine mouthwash for one week

- Give post-operative instructions

- Follow up in 7-10 days

- Perform clinical and radiographic assessments at 2 weeks, 4 weeks, 3 months, 6 months and yearly there after- monitor for signs and symptoms of loss of vitality (tooth non-responsive to vitality testing, tender to percuss, change of colour, sign of draining sinus).

\section{Avulsed Permanent Incisor - \\ Closed Apex}

For closed apex reimplantation instructions are the same as per open apex above however at 7-10 days post reimplantation root canal treatment must be commenced.

- 7-10 days post reimplantation

- Place calcium hydroxide as intra-canal medicament for up to one month

- Follow with suitable root canal filling material

- Splint removal at 2 weeks as per open apex
- Follow up at 2 weeks, 4 weeks, 3 months, 6 months and yearly there after as per open apex.

Patient advice post-reimplantation Patients should:

- Avoid contact sport

- Have a soft diet for two weeks

- Brush their teeth with a soft toothbrush

- Use chlorohexidine mouthwash two times a day for one week

\section{Dento-alveolar Splint Fabrication}

Non-rigid splinting

Here are a few tips to ensure you get your splint right:

- Adjust the length of the wire so it extends one to two teeth on either side of repositioned tooth

- Reposition the tooth

- Prophy the enamel with pumice

- Apply etchant and bonding solution

- Place a dab of composite in the centre of the facial surface of the tooth to be bonded

- Position the wire on to the composite

- Set the composite

- Add additional composite so the wire is covered, especially at the distal terminal ends

- Smooth the composite to ensure that there are no rough surfaces that might irritate the soft tissue

- Prescribe chlorhexidine mouthwash for one week.

Good luck!

Laura Crawford

https://doi.org/10.1038/s41406-020-0142-x 